Relations industrielles

Industrial Relations

\title{
Analyse socio-économique d'une grève, par Jean Mehling. Collection " Problèmes économiques contemporains ". Les Presses de l'Ecole des hautes études commerciales; Les Editions de la Librairie Beauchemin. Montréal. 1963. 216 pages.
}

\section{Gérard Dion}

Volume 19, numéro 1, janvier 1964

URI : https://id.erudit.org/iderudit/1021391ar

DOI : https://doi.org/10.7202/1021391ar

Aller au sommaire du numéro

\section{Éditeur(s)}

Département des relations industrielles de l’Université Laval

ISSN

0034-379X (imprimé)

1703-8138 (numérique)

Découvrir la revue

Citer ce compte rendu

Dion, G. (1964). Compte rendu de [Analyse socio-économique d'une grève, par Jean Mehling. Collection " Problèmes économiques contemporains ». Les Presses de l'Ecole des hautes études commerciales; Les Editions de la Librairie Beauchemin. Montréal. 1963. 216 pages.] Relations industrielles / Industrial Relations, 19(1), 138-139. https://doi.org/10.7202/1021391ar

Tous droits réservés @ Département des relations industrielles de l’Université Laval, 1964
Ce document est protégé par la loi sur le droit d'auteur. L'utilisation des services d'Érudit (y compris la reproduction) est assujettie à sa politique d'utilisation que vous pouvez consulter en ligne.

https://apropos.erudit.org/fr/usagers/politique-dutilisation/ 
sur le climat qui a précédé la grève du textile. Certains a-parte de l'auteur dans son récit sont aussi agaçants que déplacés L'évènement étudié dans ce chapitre méritait un meilleur traitement. L'auteur, malgré certains reproches qu'on peut lui faire, o été plus heureux dans le récit de la grève de Louiseville. Mais encore ici, c'est la «méthode de travail «Rumilly 》: I'utilisation abusive des découpures de journaux de l'époque.

Il est évident que celui qui n'a pas vécu ces grèves, comme celui qui ne cherche pas une analyse fouillée avec des préoccupations scientifiques, lira ces pages d'un autre oeil. II sera sûrement intéressé, tout autant intéressé que nous l'avons été nous-même, car c'est un ouvrage qui vaut la peine d'être lu. II ne bondira pas devant certaines interprétations fantaisistes et ne sera pas frustré par certaines omissions. Mais s'il s'attend d'y trouver l'objectivité, il ne la rencontrera pas également dans tous les chapitres.

Dans l'ensemble, c'est une oeuvre engagée. On le comprend et I'accepte. Une telle littérature a sa place et son utilité. A condition qu'elle se présente sous son vrai jour. Voild̀ pourquoi, nous nous réjouissons de la publication de cet ouvrage. Comme tel, il rendra service à plusieurs pour leur faire comprendre un peu certains aspects de la vie d'une centrale ouvrière et les difficultés auxquelles se sont buttés les travailleurs pour avoir droit de cité dons notre province. II montrera aussi comment nous ovons besoin d'études de recherches et publications dans un domaine aussi important. Nous espérons que des professionnels se mettront à la tâche pour combler ce vide.

\section{Gérard Dion}

Analyse socio-économique d'une grève par Jean Mehling. Collection \&Problèmes économiques contemporains ». Les Presses de I'Ecole des hautes études commerciales; Les Editions de la Librairie Beauchemin. Montréal. 1963. 216 poges.

A la fin de la recension que je faisais de l'ouvrage En Grève publié en collaboration aux Editions du Jour, j'exprimais le désir de voir se constituer chez nous une littérature sur pareils sujets, mais qui serait le fruit du travail de professionnels. Je ne pensais pas avoir d'occasion d'apprécier aussi tôt la valeur d'un tel voeu et ses limitations. Après ovoir lu Analyse socio-économique d'une grève du professeur Jean Mehling, j'ai l'impression que cet ouvrage ne réussira pas à intéresser outant de lecteurs que le précédent. Ceux-ci n'auront peut-être pas entièrement raison, mais je les comprendrai facilement.

Analyse socio-économique d'une grève veut être, comme son titre l'indique, une tentative d'étudier sous ces deux aspects la grève que les Métallos-Unis d'Amérique ont soutenu contre la Noranda Mines du 22 août 1953 au 13 février 1954. Dans l'histoire des relations patronales-ouvrières du Québec, cette grève est un évènement importont et significatif. L'ouvrage est divisé en cinq chapitres: lo grève dans son contexte historique et conjoncturel; le rôle de la Compognie dans le développement des activités locales ou le «cadre local 》 de la grève et la mobilité de la main-d'oeuvre; la capacité de résistance de la Compagnie en face de la grève; la capacité de résistance des travailleurs; coût de la grève et capacité de résistance à long terme.

L'outeur procède d'une façon méthodique. II s'est donné la peine de bâtir un modèle d'analyse économique qu'il essaie d'appliquer au cas étudié. Les réponses aux questions qu'il se pose sont assez décevantes. Parfois, ce fut à cause d'absence de données précises qu'il $n^{\prime}$ o pu se procurer. (Je m'étonne cependant qu'il n'ait pos pu mettre la main sur le dossier complet de l'arbitrage qui a précédé la grève et qui se trouve sûrement au Ministère du travail à Québec. Cela I'aurait éclairé davantage sur des foits et des attitudes qui expliquent les évènements ultérieurs). En d'autres cas, ce fut oussi pour la simple raison que certaines questions - on le sait déjà depuis longtemps - sont sons réponse. D'ailleurs l'intuition des travailleurs et de leurs dirigeants (cela est vrai oussi pour les chefs d'entreprises, mais avec beaucoup moins de risque), est parfois aussi valable que les conclusions scientifiques qui dépendent de tellement d'hypothèses non vérifiées et non vérifiables qu'à la fin elles sont inutilisables.

Le reproche le plus sérieux qui pourrait être fait à cette étude, c'est que l'auteur essaie de tout quantifier au niveau de la microéconomique, alors qu'une grève, comme celle de Noranda et presque toute grève, s'insère à l'intérieur de tout un contexte psychosocial de politique syndicale où il est impossibie de mettre en balance un avantage monétaire ovec une satisfaction psychologique. L'outeur semble avoir oublié en cours de route que la grève possède une fonction de défoulement devant une situation que les trovailleurs et leurs dirigeants ne peuvent plus endurer. La logique de l'action n'obéit 
pas aux mêmes lois que la logique des calculs strictement comptables.

Cet ouvrage est bien fait pour ceux qui aiment à jouer avec des concepts, des hypothèses. Por des cheminements longs et ardus, ou bien il n'arrive à peu de chose, ou bien il aboutit à quelques conclusions valables qui sont de sens commun et très familières à tous ceux qui ont une expérience syndicale. Por ailleurs certaines de ses assertions sont plus que discutables. En voici un exemple: \&ll est difficile d'imaginer que la grève, échec pour les travailleurs considérés à titre individuel, soit une victoire pour le Syndicat, entité différente de ses membres \$. (p. 174) Si cela peut être vrai en courte période, il est assez facile de concevoir qu'en longue période il n'en est pos nécessairement ainsi.

L'ouvrage du professeur Mehling est remarquable par son souci d'objectivité. II n'a rien $d^{\prime}$ anti-syndical, mais il fera les délices de ceux qui, ne comprenant rien à l'action syndicale, trouvent toujours que ces groupements sont trop enclins à se lancer dans des oventures préjudiciables à leurs membres et à toute la communauté. J'hésiterais à le mettre entre les mains d'un dirigeant syndical qui aurait la patience de le prendre à la lettre et de le lire jusqu'au bout. Car, devant la multitude de questions qui restent sans réponse et l'incertitude des résultots dans une grève, ou bien ce dirigeant demeurerait toujours dans la perplexité et abandonnerait les travailleurs au bon vouloir des employeurs puissonts qui s'ocharneraient d̀ les tenir dans une situation d'injustice, ou bien il s'organiserait pour inciter les travailleurs à une action d'une telle violence que la grève ne serait pas de longue durée. $\mathrm{Ce}$ n'est certainement pas l'intention poursuivie par l'auteur.

Analyse socio-économique d'une grève est un livre utile. Préporé par un économiste sérieux et compétent, il illustre bien les difficultés que rencontre toute étude de ce phénomène économico-social qu'est la grève.

\section{L'Entreprise et la Statistique, tome 2, par} R. Dumos, Dunod, Paris, 1963, 295 poges.

II ne s'agit pas, loin de là, d'un manuel conventionnel de statistiques. L'auteur parle plutôt de l'information et de son traitement au sein de l'entreprise. II va même plus loin; i) situe certains problèmes majeurs qui se posent dans les entreprises modernes et développe des techniques d'analyse. La simple énumération des grands sujets traités nous permet de mieux voir le contenu du livre: la statistique et l'entreprise; le tableau de bord et les ratios; le contrôle des fabrications; la recherche opérotionnelle; l'étude du marché; la conjoncture; la statistique et la comptabilité; I'organisation d'un service statistique d'entreprise; des études de cas.

On pourrait dire que ce volume est une sorte d'introduction à divers sujets de l'administration de l'entreprise. L'auteur insiste toutefois sur les statistiques nécessaires à l'étude de ces sujets. Tel n'est pas le cas pour tous les sujets. La recherche opérotionnelle, por exemple, est analysée dans sa méthodologie même plutôt qu'en tant que statistiques à incorporer dans des modèles mathématiques; il s'ogit donc en l'occurence d'une sorte de très brève introduction da la recherche opérationnelle. Au contraire dans l'étude du marché, l'auteur ne fait pas seulement présenter une méthode de travail, mais mentionne plusieurs sortes de statistiques qui sont incluses dans de telles études.

Qui ce livre peut-il intéresser? Nous croyons que les hommes d'affaires soucieux d'améliorer la gestion de leurs entreprises pourraient retirer profit de la lecture de «L'Entreprise et la Statistique ». Nous en conseillons également la lecture aux administrateurs qui ont besoin de connaître les différentes techniques d'analyse des problèmes des entreprises. Nous ne croyons pas qu'il puisse intéresser les spécialistes de la gestion, car l'exposé des divers sujels ne va pas assez en profondeur.

Bertrand Belzile

\section{RELATIONS INDUSTRIELLES}

volume 18

\section{S O M M A I R E}

Index bibliographique - Cumulative Index. REVUE Relations industrielles Industrial Relations, REVIEW volume 1-18 (1945-1963) Rapports des Proceedings of Congrès des Relations industrielles (1946-1963) Index Volume 18 - 1963. 